\title{
LOS CÍRCULOS DE CALIDAD Y SUS EFECTOS EN LA ETAPA DE PLANEAMIENTO DURANTE LA GESTIÓN DE LAS UNIVERSIDADES PRIVADAS EN LIMA METROPOLITANA
}

\author{
Charles Pastor Torres Vásquez* \\ charlestorres15@yahoo.com
}

\begin{abstract}
RESUMEN
La calidad es hoy en día sinónimo de satisfacción del cliente. El problema se observa en que las universidades privadas de Lima Metropolitana tienen la necesidad de aplicar, en el planeamiento, círculos de calidad, para contribuir a optimizar, su gestión.

Los materiales y métodos que se utilizaron para desarrollar la tesis fueron información de libros, revistas e internet, teniendo la hipótesis y los objetivos del presente trabajo, éste se ubicará en el paradigma mixto; cuantitativo - cualitativo.

Para resolver el problema, se aplica los círculos de calidad y las concepciones modernas de empoderamiento administrativo y la identificación del nivel correlacionar que existe con el planeamiento en las gestiones universitarias.

Como resultado, ha quedado demostrado que la organización de los círculos de calidad crea conciencia de calidad y productividad en cada uno de los miembros de una institución, a través del trabajo en equipo, contribuyendo a optimizar el planeamiento basado en la observación del futuro; al recomendar estrategias y políticas en beneficio de las universidades privadas de Lima Metropolitana.

Palabras clave: Planificación, Círculos de calidad, Gestión.

\section{ABSTRACT}

Quality becomes of nowadays synonymous the customer's satisfaction. The problem is that Universities in Lima need to apply a planning of quality circles in order to optimize its management. It was used as method a quantitative - qualitative paradigm, using books, magazines and the Internet sources of information. To resolve the problem, quality circles and modern conceptions of administration empowerment were used to have a correlation in order to know the planning of universities management. As a result, it is been confirmed that quality circles creates a quality and productivity competences in members of an institution, through an equipment. This way of work optimizes planning based on future needs: strategies and politics in behalf of the University in Lima.
\end{abstract}

Keywords: planning, circles of quality, management

\footnotetext{
* Doctor en Educación, Doctorando en Administración (UNMSM), Magister en proyectos y Desarrollo de Empresas, Magíster en Gestión y Desarrollo. Magíster en Docencia Universitaria, Licenciado en Administración. Licenciado en Educación Secundaria, Licenciado en Ciencias del Deporte. Profesor de Pos Grado: UNMSM, UNPRG, UIGV. ICTE. Docente Investigador. Director de la Escuela de Pos grado del ICTE.
} 


\section{INTRODUCCIÓN}

Las instituciones en nuestro país se encuentran en una etapa de cambios significativos o importantes debido a la globalización en las que nos encontramos inmersos. Las empresas más fuertes sobreviven, las que no, simplemente desaparecen del mercado a la cual pertenecen. Algunas empresas utilizan estrategias para "sobrevivir", pero quizá la más importante es la de brindar calidad como: calidad en el servicio, de producto, de atención, etc.; lamentablemente, pocas empresas ofrecen esta característica, ya sean privadas o estatales.

Si nos centramos básicamente en universidades que forman parte del sistema privado, nos encontramos con la misma realidad; es decir, estos adolecen de los mismos problemas como las demás salvo excepciones, como por ejemplo mantenimiento, presupuesto, servicio de calidad, capacitación, etc. Es de suponer que siendo casas superiores de estudio, la carencia física de estos elementos es superada por el conocimiento donde la teoría que se pregona es puesta en práctica dentro y fuera de sus fronteras. La gestión de las universidades muchas veces tiene un alto grado de ideología, intereses políticos y económicos, como se ve y escucha actualmente.

Las universidades son las encargadas de definir modelos organizacionales coherentes y validadas por gestión, evaluación y seguimiento constante de la calidad de los resultados, donde los estudiantes forman la materia prima de la mejor calidad, de tal forma que las empresas del sector privado o estatal sean las primeras en solicitar los servicios profesionales de los egresados, por supuesto este producto es el resultado del planeamiento que en cierta medida han hecho sus autoridades académicas.

El planeamiento es un proceso mediante el cual los miembros guías de una organización, que en este caso lo conforman el vicerrector Administrativo, académico o el rector, prevén el futuro organizacional y desarrollan los procedimientos y operaciones necesarias para alcanzarlo, siendo, por lo tanto, un factor importante para lograr los objetivos que toda empresa u organización plantea, como el caso de las universidades.

Por otro lado, se considera que no existe un buen plan sin antes existir un adecuado planeamiento de lo que se quiere. En algunas universidades se viene implementando los denominados círculos de calidad para realizar un buen pla- neamiento, pues es sabido que los elementos o trabajadores que conforman el problema son los mejores elementos para solucionarlos pues la gente se internaliza en sus respectivos puestos de trabajo y conocen lo que sucede, lo que les falta o sobra. El planeamiento está relacionado también con los círculos de calidad porque trata de reducir los errores y aumentar la calidad, además incrementa la motivación, crea actitudes para prevenir problemas y capacidad para resolverlos, y promueve el desarrollo personal.

Por lo tanto, los círculos de calidad y el planeamiento juegan un rol importante en la consecución de los objetivos organizacionales, razón por la cual es necesario conocer si las universidades privadas realmente aplican total o parcialmente algunos conceptos de los círculos de calidad y realizan el planeamiento adecuadamente.

En ese sentido, el problema a investigar se centra en la forma en que están organizados los círculos de calidad y sus efectos en el planeamiento en las universidades privadas.

El problema descrito en párrafos anteriores se manifiesta de manera compleja, sin embargo, por ser de interés del autor la presente investigación se realizará de manera empírica y por observación no comprobada, piensa que el problema se verificará o negará en el diagnóstico, se manifiesta de manera compleja de interés para el presente trabajo, solo se referirá a dos para la presente tesis:

1. Círculos de calidad.

2. Optimizar el planeamiento de las universidades.

Tiene como objetivo principal analizar si la organización de los círculos de calidad contribuye a optimizar la gestión de las Universidades Privadas de Lima Metropolitana.

La presente investigación se justifica, al tener la necesidad de conocer y reconocer el nivel de implementación de los círculos de calidad en el cumplimiento de los objetivos, como una herramienta eficaz de gestión administrativa; el reconocimiento de la organización y aplicación de estrategias que integren diversas áreas de la Universidad, bajo una única visión, que el planeamiento tome acciones adecuadas hoy, que proporcione los medios básicos para dirigir el comportamiento de la Universidad como organización; planteando un marco teórico referencial, para la gestión administrativa 


\section{MÉTODOS}

Por el tipo de investigación es una investigación aplicada pues busca establecer una serie de proposiciones coherentes sobre un objeto de estudio. Teniendo la hipótesis y los objetivos del presente trabajo, este se ubicará en el paradigma mixto; cuantitativo - cualitativo.

Considerando que el diseño de investigación es el plan de acción, indica la secuencia de los pasos a seguir que le permitirán al investigador precisar los detalles de las tareas de investigación y establecer las estrategias a seguir para obtener resultados positivos, además de definir la forma de encontrar las respuestas a las interrogantes que inducen al estudio.

La población (N) objeto de estudio estuvo conformada por 17 rectores de las universidades privadas de Lima Metropolitana, las que se detallan a continuación en la parte inferior. Esta información fue obtenida de la página Web de la Asamblea Nacional de Rectores (www.anr.edu. pe).: Universidad Peruana de Ciencias Aplicadas; Universidad Particular Marcelino Champagnat; Universidad Peruana Unión; Universidad Ricardo Palma; Universidad de Piura; Pontificia Universidad Católica de Lima Metropolitana; Universidad de San Martín de Porres; Universidad Inca Garcilaso de la Vega; Universidad de Lima; Universidad del Pacifico; Universidad Peruana Cayetano Heredia; Universidad Tecnológica del Perú; Universidad Privada "San Ignacio de Loyola"; Universidad Norbert Wienner; Universidad Alas Peruanas; Universidad TELESUP y Universidad de Ciencias Aplicadas. Para seleccionar la muestra se utilizó la técnica de selección aleatoria; y el instrumento de recolección de datos mediante el análisis documental y la encuesta.

Por lo tanto el entorno de la investigación se sitúa en las diferentes universidades previamente detalladas.

En el análisis estadístico se desarrolló con el gráfico de GANTT y el método del camino crítico. Este gráfico cubre las distintas funciones de la programación: asignación de recursos y determinación de la secuencia y la cronología. A pesar de su simplicidad, constituye una de las herramientas de programación más completas y, sin duda, la más usada.

Es así que el gráfico se confecciona a dos escalas: en la horizontal se mide el tiempo; y en la vertical se ordenan los elementos que intervienen en la programación. El gráfico establece, de tal forma, una correlación cronológica entre los distintos recursos o tareas.

Las subdivisiones horizontales del espacio en el gráfico representan a su vez tres cosas: transcurso de una unidad de tiempo, trabajo programado para ese intervalo y trabajo realizado efectivamente en el mismo. La inclusión simultánea de estos dos últimos aspectos implica cotejar lo programado con lo realizado, es decir, controlar el cumplimiento del programa.

\section{DISEÑO GRÁFICO:}

$$
\mathbf{n}=\mathbf{O x} \Rightarrow \mathrm{r} \text { OY }
$$

Donde:

$\mathrm{n}=$ Muestra.

$\mathrm{O}=$ Observación

$\mathrm{x}$ = Organización de los Círculos de Calidad.

y = Optimización en el Planeamiento.

$\mathrm{r} \Rightarrow=$ Relación de variables

\section{Resultados:}

Los datos han sido clasificados en una tabla de contingencia para probar la hipótesis, de independencia de criterios, mediante la Probabilidad Exacta de Fisher.

H0: La organización de los círculos de calidad contribuye a optimizar el planeamiento de las Universidades Privadas de Lima Metropolitana.

H1: La organización de los círculos de calidad contribuye a optimizar el planeamiento de las Universidades Privadas de Lima Metropolitana.

\begin{tabular}{|l|c|c|c|c|}
\hline \multirow{2}{*}{$\begin{array}{l}\text { Nivel de organi- } \\
\text { zación de los cír- } \\
\text { culos de calidad }\end{array}$} & \multicolumn{3}{|c|}{$\begin{array}{c}\text { Realizan planeamiento en las } \\
\text { Universidades Privadas }\end{array}$} & \multirow{2}{*}{ Total } \\
\cline { 2 - 4 } & Totalmente & Parcialmente & Nada & \\
\hline Excelente & 3 & 1 & 1 & 5 \\
Buena & 0 & 8 & 0 & 8 \\
Regular & 1 & 1 & 0 & 2 \\
\hline Total & 4 & 10 & 1 & 15 \\
\hline
\end{tabular}

Para probar la hipótesis planteada seguiremos el siguiente procedimiento:

1. Nivel de significación: $\alpha=0.05 \mathrm{y} \mathrm{n}=15$

2. Región de rechazo: Ho será rechazada si los valores observados difieren en la dirección 
predicha y si son de tal magnitud que la probabilidad asociada con su ocurrencia conforme a Ho es igual o menor a $2^{\alpha}=0.1$.

3. Prueba estadística.

$P(f)=\frac{(A+B !)(C+D !)(A+C !)(B+D !)}{N ! A ! B ! C ! D !}=0.0732$

4. Decisión: La información concerniente a las variables se encuentra clasificados en la tabla anterior, cuyo valor de la probabilidad exacta de Fisher es inferior a $2 \alpha$ por lo que la hipótesis nula se rechaza.

5. Conclusión: La organización de los círculos de calidad contribuye a optimizar el planeamiento de las Universidades Privadas de Lima Metropolitana.

\section{DISCUSIÓN}

Un aspecto importante dentro de todo circulo de calidad es el consenso que puedan lograr los miembros que la componen, en especial cuando se trata de tomar una decisión que tenga trascendencia, en ese sentido, el $60 \%$ de los encuestados, señalaron mantener siempre consenso en bien de la universidad y tomar la decisión conveniente, manifestaron unirse y realizar consenso ya que las decisiones que se puedan tomar tienen que ir de acuerdo al criterio común y no responder a voluntades de tipo político o simplemente para beneficio personal; esto quiere decir que cuando hay consenso hay beneficio para la institución universitaria.

Existen diversas maneras de optimizar el trabajo como parte del planteamiento una de ellas es organizar los objetivos de trabajo con diversas estrategias, aquí también la estrategia más adecuada es la que permite ahorro de tiempo y el mejor manejo de la información es precisamente con la tecnología de la información que según el $80 \%$ de los encuestados manifestaron utilizarla siempre, por otro lado el $20 \%$ restante manifestó hacerlo a veces pues es indudable que este pequeño porcentaje aún no maneja adecuadamente la tecnología de información.

\section{CONCLUSIONES}

1. Ha quedado demostrado, que el nivel de consenso en el grupo que conforma los círculos de calidad; permite establecer políticas y estrategias idóneas en beneficio de la Universidad.
2. Se ha determinado y demostrado que la reducción de errores y por ende el aumento de la calidad, como resultante de una correcta administración eficiente de los recursos y presupuestos disponibles; coadyuvan a incrementar la productividad de la administración de los presupuestos en beneficio de la Universidad.

3. Ha quedado determinado que el descubrimiento e identificación de problemas y sus respectivas soluciones, establecen una secuencia coherente de las acciones a realizar; con el fin de lograr una situación viable y original, orientado a mejorar la conducción de la Universidad; así como anticipando los posibles cambios en el entorno y las acciones imprevistas de los oponentes inteligentes.

4. Ha quedado demostrado que el nivel de colaboración entre participantes, organizado y conducido en base al liderazgo, la participación, la mejora de los ambientes laborales y un aumento de la moral; permite interrelacionar diferentes Áreas de la Universidad, contando con valores, habilidades y conocimientos; para la transformación y el desarrollo de la casa superior de estudios.

5. Como conclusión final, ha quedado demostrado que la organización de los círculos de calidad; crea conciencia de calidad y productividad en cada uno de los miembros de una institución, a través del trabajo en equipo y el intercambio de experiencia y conocimiento, contribuyendo a optimizar el planeamiento basada en la observación del futuro; al recomendar estrategias y políticas en beneficio de las Universidades Privadas de Lima Metropolitana.

\section{REFERENCIAS BIBLIOGRÁFICAS}

ACEVEDO, JORGE. LA SOCIEDAD COMO PROYECTO - "MODERNIDAD Y POSTMODERNIDAD. (VIDA PERSONAL E HISTORIA: UN RETORNO HACIA LO CERCANO)" - ; Editorial Universitaria, Santiago, 1994. Capítulo III, pp. 103.

ACKOFF, Rusell L. CREANDO LA CORPORACIÓN DEL FUTURO, Editorial John Wiley \& Sons, primera edición, Nueva York-Estados Unidos, 1981, pp. 462.

EMERY, James L. SISTEMAS DE PLANEAMIENTO Y CONTROL EN LA EMPRESA, Editorial El Ateneo, primera edición, Buenos AiresArgentina, 1973, pp. 378. 
EPPEN, G. D.; GOULD, F.J.; SCHMIDT, C. P.; MOORE, J. H. y WEATHERFORD, L.R.. INVESTIGACIÓN DE OPERACIONES EN LA CIENCIA ADMINISTRATIVA, Editorial Prentice Hall Hispanoamericana S.A., quinta edición, México, 2000, pp. 702.

FERNÁNDEZ VALDERRAMA, Juan Carlos. SISTEMAS DE PARTICIPACIÓN. CÍRCULOS DE CALIDAD, Editorial ICESI, primera edición, Colombia, 2001, pp. 175.

FLIPPO, Edwin B. y MUNSINGER, Gary M. DIRECCIÓN DE EMPRESAS, Editorial El Ateneo, tercera edición, Buenos Aires-Argentina, 1984, pp. 658.

GÓNZALES OUBEL, M.. LOS CÍRCULOS DE CALIDAD, UN PROCESO DE DESARROLLO DE LA DIRECCIÓN, Editorial Alta Dirección, 1987, pp.

GONZÁLEZ ROMA, V. UN ANÁLISIS DE LA PARTICIPACIÓN EN EL TRABAJO Y LOS CÍRCULOS DE CALIDAD, Editorial de Psicología del trabajo y de las organizaciones, décima edición, 1992, pp. 197.

HERNÁNDEZ Roberto, FERNÁNDEZ Carlos, BAPTISTA Pilar Lucio, METODOLOGÍA DE LA INVESTIGACIÓN CIENTÍFICA, Editorial Mc. Graw Hill. México, 2000, pp. 501.

HERNÁNDEZ, FERNÁNDEZ Y BAPTISTA. METODOLOGÍA DE LA INVESTIGACIÓN CIENTÍFICA. México.

HISTORIA DEL PENSAMIENTO LECTURAS 1. , Universidad Panamericana, México, D. F. Agosto 2000.

IBAÑEZ MACHICAO, Mario. CALIDAD TOTAL. RETO EMPRESARIAL, Editorial Consejo Nacional de Ciencia y Tecnología - CONCYTEC, Lima-Perú, 1996, pp. 232.

KRAMSKY STEINPREIS, Carlos. ANTROPOLOGÍA FILOSÓFICA., Editorial Murcia 16-301, México DF. 1997. pp. 310.

LEVIONNOIS, M. MARKETING INTERNO Y GESTIÓN DE RECURSOS HUMANOS, Editorial Díaz de Santos, segunda edición, MadridEspaña, 1992, pp. 325

MARTÍNEZ MUT, B.. TECNOLOGÍA DEL ANÁLISIS DE PROBLEMAS EN LOS CÍRCULOS DE CA-
LIDAD, Editorial Interuniversitaria, primera edición, España, 1986, pp. 156

MORENO LUZÓN, M. D. EL TRABAJO EN EQUIPO Y LA CALIDAD TOTAL, Editorial ESIC-MARKET, cuarta edición, España, 1995, pp. 427.

ORDÓÑEZ ACOSTA, J. LOS CÍRCULOS DE CALIDAD Y SU INFLUENCIA EN LA GESTIÓN DE LA EMPRESA, Editorial Alta Dirección, primera edición, España, 1989, pp. 564.

PALOM IZQUIERDO, F. J. CÍRCULOS DE CALIDAD: TEORÍA Y PRÁCTICA, Editorial Marcombo, segunda edición, Barcelona-España, 1987, pp. 418.

RESBIER, E. y ALMIRALL, M. LOS CÍRCULOS DE COOPERACIÓN, Editorial Fundación Joan Ventosá i Roig, primera edición, BarcelonaEspaña, 1989, pp. 312.

REVISTA DE FILOSOFÍA VOL. XIII, N' 1 “LA CONSTITUCIÓN ONTO-TEO-LÓGICA DE LA METAFÍSICA", Santiago, 1966; trad. de Luis Hernández, revisada por Francisco Soler; p. 100

RODRÍGUEZ PORRAS, J. M. LA PARTICIPACIÓN Y LA CALIDAD INTEGRAL, Editorial Deusto, primera edición, Bilbao, 1992, pp. 352.

SADERRA JORBA, LL. LA CALIDAD TOTAL, Editorial Técnica Rede, tercera edición, BarcelonaEspaña, 1994, pp. 322

SIDNEY Siegel. , ESTADÍSTICA NO PARAMÉTRICA APLICADA A LAS CIENCIAS DE LA CONDUCTA, Editorial Trillar S.A., Segunda Reimpresión en Español, México 1 D.F. 1972.

TERRY, George R. y FRANKLIN, Stephen G.. PRINCIPIOS DE ADMINISTRACIÓN, Compañía Editorial Continental S.A. de C.V., Décima Cuarta Reimpresión, México D.F., 1999, pp. 747.

URIZAR, E. METODOLOGÍAS DE CALIDAD TOTAL PARA PERSONAL TOTAL, Editorial Fundación Vasca para el Fomento de la Calidad, primera edición, España, 1997, pp. 269.

WANTY, Jacques y HARBERTHAL, Isi. LA ESTRATEGIA EMPRESARIAL, Editorial El Ateneo, primera edición, Buenos Aires-Argentina, 1975, pp. 395. 